\title{
Reasentamiento involuntario: políticas y prácticas en los Andes
}

Involuntary resettlement: policy and practices in the Andes

\section{Gerardo Damonte}

Grupo de Análisis para el Desarrollo, Pontificia Universidad Católica del Perú, Departamento de Ciencias Sociales, Perú gdamonte@grade.org.pe

Manuel Glave

Grupo de Análisis para el Desarrollo, Pontificia Universidad Católica del Perú, Departamento de Ciencias Sociales, Perú

mglave@grade.org.pe

\section{ReSUMEN:}

En las últimas décadas, se han desarrollado políticas internacionales para guiar los procesos de Reubicación Involuntaria con el fin de garantizar la reconstrucción social y mejoras en los estándares de vida de las comunidades reasentadas; sin embargo, las experiencias andinas nos muestran que, en general, estos objetivos no han sido alcanzados. ¿Cómo ajustar el diseño de políticas globales de reasentamiento involuntario para una mejor implementación en los Andes? Este artículo, siguiendo enfoques de desarrollo localmente situados, argumenta que las políticas de reasentamiento involuntario no se han ajustado adecuadamente al contexto y la cultura andina, lo que generó malas interpretaciones culturales y conflictos que a su vez han impedido su cumplimiento. El artículo se basa en el análisis del proceso de reubicación llevado a cabo por la compañía minera Antamina en la comunidad peruana de Yanacancha.

Palabras Clave: Reasentamiento involuntario, Minería, Andes, Pastores, Banco Mundial.

\section{Abstract:}

In recent decades, international policies have been developed to guide processes of involuntary resettlement in order to guarantee social reconstruction and improvements in the living standards of resettled communities; however, Andean experiences show us that, in general, these objectives have not been achieved. How to adjust the design of global involuntary resettlement policies for better implementation in the Andes? This article, following Place-Based Development perspectives, argues that the policies of involuntary resettlement have not adapted adequately to the Andean context and culture, generating bad cultural interpretations and conflicts that in turn have prevented its fulfillment. The article analyzes the relocation process carried out by the mining company Antamina in the Peruvian community of Yanacancha.

KEYWORDs: Involuntary resettlement, Mining, Andes, Pastoralism, World Bank.

\section{INTRODUCCIÓN}

El desarrollo de grandes proyectos de minería ha implicado el desplazamiento de las poblaciones que han ocupado y usufructuado el territorio donde se construye la operación minera. Sin embargo, estos desplazamientos no suelen llevarse a cabo siguiendo procesos participativos donde los afectados puedan expresar su opinión informada. Las empresas, típicamente, han desplegado mecanismos legales disponibles para compeler o coaccionar la transferencia de tierra y el desplazamiento poblacional. La literatura disponible muestra que la simple adherencia a los derechos legales de los desplazados, tal como son definidos por el Estado, así como el pago del precio de mercado de la tierra, han demostrado ser de poca influencia en los 
resultados esperados (McDowell, 1996; Cernea, 2000). Las poblaciones desplazadas no solamente pierden el poder sobre sus propios medios de vida, sino que también, en la mayoría de los casos, se hacen más pobres como resultado.

En respuesta a esta problemática, organismos multilaterales como el Banco Mundial han desarrollado lineamientos de política sobre reasentamiento involuntario, tales como la Política Operacional 4.12 del Banco Mundial (OP 4.12) o el Estándar de Desempeño \# 5 de la Corporación Financiera Internacional (IFC PS 5), cuyo objetivo central es asegurar la reconstrucción social y la mejora de vida de las poblaciones reubicadas. En el Perú este hecho ha sido particularmente importante puesto que ha generado un marco de política, ante la ausencia de normativas y regulaciones estatales sobre planes de reasentamiento en proyectos mineros (salvo las que se contemplan en el Plan de Manejo de los Estudios de Impacto Ambiental). Sin embargo, la literatura mayormente encuentra que la aplicación de esta normativa en los Andes ha sido disímil, conflictiva y poco exitosa. Por un lado, los estudios han analizado la complejidad e idoneidad legal; así, las leyes nacionales se entrecruzan y entran en conflicto con normatividades corporativas transnacionales (Szablowski, 2002; Szablowski 2007; Escobedo, 2008). Por otro lado, trabajos de corte etnográfico ponen énfasis en los conflictos y formas culturales no tomadas en cuenta en los procesos de desplazamiento y reubicación (Salas, 2008; Hervé, 2013; León, 2015; Miranda, 2016).

Este artículo busca contribuir con esta literatura desde un punto de partida distinto: el análisis de la política misma y sus vacíos al momento de la implementación. En particular, nos preguntamos ¿cómo mejorar el diseño de las políticas globales de reasentamiento involuntario para su implementación en los Andes? Para responder esta pregunta, hacemos un análisis de los problemas de implementación que observamos en el proceso de reasentamiento involuntario de la comunidad de Yanacancha, ubicada en el departamento de Ancash- Perú, por el proyecto de gran minería Antamina, con énfasis en dos temas relacionados: cómo abordar y comprender los impactos del reasentamiento y cómo asistir y monitorear el proceso de reconstrucción en comunidades rurales andinas. Nuestro argumento general es que las políticas de reubicación no han sido adecuadamente ajustadas al contexto y cultura andinas, por lo que su aplicación genera los conflictos y desfase cultural, identificados en los trabajos etnográficos, en vez de coadyuvar a la reconstrucción social de las poblaciones reubicadas. En última instancia, el artículo busca identificar las condiciones necesarias para aproximarse al cumplimiento de los estándares, utilizando como instrumento de validación el análisis de los factores que condujeron al fracaso del proceso de reubicación de Yanacancha en el proyecto Antamina.

La literatura sobre desarrollo discute las ventajas y los límites de la implementación de las políticas globales. Con la globalización, se ha vuelto muy común la transferencia de políticas públicas generadas desde los organismos internacionales hacia distintos países y/o regiones subnacionales del planeta. En resumen, se discute la viabilidad de transferir políticas de manera homogénea y la necesidad de ajustar la implementación de dichas políticas a los contextos políticos, económicos y políticos específicos (Massey, 2009; Syväterä, 2016; Barca, McCann y Rodríguez-Pose, 2012).

En este contexto, ha surgido la idea de "desarrollo localmente situado" (Place-based development) como la nueva perspectiva llamada a guiar el ajuste territorial de las políticas globales (Barca et. al., 2012; OECD, 2011). En particular, esta perspectiva busca establecer mecanismos de política pública que ayuden bajar a tierra principios de las políticas globales a contextos específicos de cada país o región. Sin embargo, esta perspectiva todavía no se ha desarrollado para las políticas globales de reasentamiento involuntario. Si bien las grandes empresas mineras han acordado guiarse por los principios de las políticas y estándares internacionales (MMSD, 2000), no ha habido un esfuerzo por establecer mecanismos para el ajuste territorial de dichas políticas y estándares.

En el caso de los procesos de reubicación por minería en los Andes, el desafío es mayor puesto que implica no solamente ser culturalmente sensible a la heterogeneidad cultural andina sino también ajustar a unidades territoriales más pequeñas que países o regiones sub-nacionales, es decir, comunidades y poblados andinos. 
Como demuestra Escobar (2001), los grupos sociales construyen su cultura e identidad cultural en relación con los espacios territoriales que habitan, por lo que el entendimiento cultural implica conocer cómo la cultura se asienta en territorios determinados. Por ejemplo, trabajos críticos sobre la implementación de políticas de conservación en comunidades pequeñas nos muestran la necesidad de entender la sociedad no como separada del territorio sino como co-productora del mismo. La invisibilización de esta interacción lleva al fracaso de las iniciativas de conservación (Agrawal y Redford, 2009). Por ello, para ajustar mejor las políticas de reubicación deben tomar las particularidades sociales de las comunidades y su interacción con el territorio que se ven obligados a abandonar.

Metodológicamente, este artículo se basa en un estudio de caso en el que se han combinado métodos etnográficos con análisis cuantitativo. La información cualitativa fue recabada por medio de entrevistas a profundidad y grupos focales. En el caso de la información cuantitativa, el estudio se basa en el análisis de una encuesta de hogares.

Este artículo se encuentra dividido en cuatro secciones. Luego de esta introducción, en la primera se presentan las políticas del Banco Mundial sobre los temas de identificación de personas desplazadas y participación informada. En la segunda, se describe analíticamente el caso del proceso de reasentamiento de Yanacancha, centrando el análisis en los temas de identificación, compensación y participación informada de las personas afectadas. En la tercera sección, se hace un análisis de las deficiencias en la implementación de las políticas de reasentamiento y se proponen formas metodológicas para superar dichas deficiencias. Finalmente, en la cuarta sección se presentan las conclusiones.

\section{Políticas de reubicación: los dilemas del desplazamiento}

El problema del desplazamiento involuntario y reasentamiento de poblaciones ha sido abordado en la literatura académica principalmente como producto de la implementación de proyectos de desarrollo o extractivos. Los primeros se agrupan bajo el rubro de "desplazamiento inducido por desarrollo" (DIDR, por sus siglas en inglés); es decir, por la necesidad de construir infraestructura que alimente el desarrollo económico y que alivie los niveles de pobreza local. A pesar de las intenciones, estos proyectos tienen a menudo efectos perversos (Cernea, 2003), como el empobrecimiento de las poblaciones que son sujetas a reubicación.

En la literatura, esto se conoce como el "efecto reasentamiento", es decir, la pérdida de activos materiales e inmateriales: viviendas, tierras, fuentes de ingreso, acceso a recursos naturales, lugares de significación cultural, redes sociales, identidades colectivas y mecanismos de ayuda mutua (Downing, 2002: 3). Las poblaciones impactadas por procesos de reasentamiento se enfrentan frecuentemente a riesgos que, de no ser contrarrestados en un plan cuidadosamente formulado, pueden ponerlas en situación de "nueva pobreza". Antes de la emisión de las políticas del Banco Mundial, los efectos del reasentamiento no eran apreciados en su totalidad, pues el foco se ubicaba sobre los activos físicos, y se dejaban a un lado variables no monetizadas. Las familias o comunidades resarcidas eran compensadas económicamente por la pérdida de sus viviendas o tierras, pero esto no llevaba a la restitución de la calidad de vida previa. De este modo, el principal problema que generan los proyectos de reasentamiento inducido por desarrollo es el desmantelamiento de la base económica de las poblaciones a las que afecta.

Con esta perspectiva en mente, el Banco Mundial adoptó en 1980 una serie de lineamientos generales que sentaron las bases para el manejo de proyectos de desarrollo que involucren el desplazamiento de poblaciones. Esta fue revisada en 1990 para incluir políticas específicas al caso de construcción de represas. Finalmente, en 2002, se constituyó la llamada Política Operacional 4.12 (OP 4.12), que incorporó la experiencia en reasentamientos en diversos sectores de intervención a lo largo del mundo (Banco Mundial, 2004: 3). En ella se fijaron parámetros operacionales para los Estados y empresas interesadas en llevar a cabo este tipo de proyectos. El Banco Mundial puso el énfasis de la OP 4.12 en los procesos de adquisición de tierras cuyos 
resultados sean la reubicación de viviendas, pérdida de activos o acceso a activos, así como la pérdida de medios de vida (aun cuando entre los afectados no haya habido reubicación de personas de por medio).

De este modo, la evolución de las políticas del Banco Mundial estableció que las intervenciones de reasentamiento deban ser tratadas simultáneamente como proyectos de desarrollo local (Banco Mundial, 1986; Pince, 2009; Cernea, 1988: 45), que reviertan los impactos generados y se evite el empobrecimiento más allá de la compensación limitada de las pérdidas monetizables. Un importante detalle semántico debe ser precisado pues en las directivas iniciales se establecía que la meta debía ser "restaurar y en lo posible mejorar" la calidad de vida de los afectados, mientras que en la revisión de 1990 esta cambia a "mejorar o por lo menos restaurar", invirtiendo el orden de las prioridades (Cernea, 2003: 43-44). Asimismo, a partir de estos cambios, el cálculo de los costos de los proyectos de infraestructura debía incluir las pérdidas ocasionadas a la comunidad, internalizando costos que de otro modo no serían tomados en cuenta o serían abiertamente ignorados (Cernea, 1997: 1579).

El peligro más severo de un reasentamiento mal concebido consiste en que, sin entender las dinámicas sociales de la comunidad, la intervención genere inequidades y desbalances en la dinámica social existente (Salas, 2008), y exacerbe las desventajas de algunos actores y dé mayores beneficios a otros (Cernea, 2003: 37). Lo que algunos observadores y estudiosos del tema proponen como solución o remedio a estos problemas es el llamado principio de compensación, que consiste en resarcir a los afectados por desposesión y pérdida de ingresos con incentivos económicos traducidos en transferencias directas de capital o la compra compensatoria de sus activos.

Este principio, sin embargo, no es completamente satisfactorio, pues a la luz de la experiencia, la compensación se revela insuficiente para restaurar las formas de vida de los afectados por desplazamiento involuntario (Kanbur, 2003; Cernea, 2003). Así, resulta necesario repensar completamente los mecanismos de gestión social del reasentamiento y trascender una perspectiva centrada en la compensación a otra más preocupada por la mejora del ingreso de los individuos afectados para lograr su desarrollo, en lo que ha venido a denominarse el modelo de riesgo y reconstrucción (Cernea, 1997; Cernea, 2000). Cernea parte de que es necesario considerar el desplazamiento forzado como un acontecimiento externo que irrumpe en la vida de los afectados, destruyendo sistemas de producción y las dinámicas sociales que los lubrican. El desplazamiento, sin una estrategia claramente definida de reconstrucción de modos de vida y centrado únicamente en mecanismos de compensación, debilita el capital humano y social de las comunidades afectadas.

El modelo propuesto por Cernea, y recogido por las directivas del Banco Mundial, es una herramienta para generar y organizar el conocimiento obtenido durante las intervenciones en comunidades afectadas por reasentamiento, así como una guía para la acción aplicable a los procesos de planificación (Cernea, 1997: 1571). Está basado en cuatro componentes o funciones interrelacionadas: diagnóstico, investigación, predicción y resolución de problemas. A través de este modelo se han logrado identificar los ocho principales riesgos de empobrecimiento a los que las familias y comunidades afectadas se enfrentan durante los procesos de reasentamiento involuntario: (1) pérdida de tierra, (2) pérdida de empleo, (3) pérdida de hogar, (4) marginalización, (5) aumento de morbilidad y mortalidad, (6) inseguridad alimentaria, (7) pérdida de acceso a la propiedad comunal, (8) desarticulación social.

La Política Operacional 4.12 del Banco Mundial sobre Reasentamiento Involuntario (en adelante se referirá a ella como la politica) es una respuesta a este problema. Es una política obligatoria aplicable a todos los proyectos que reciben financiamiento del Banco Mundial, incluyendo aquellos proyectos del sector privado asistidos por la Corporación Financiera Internacional (IFC) o el Organismo Multilateral de Garantía de Inversiones (MIGA). La Política Operacional 4.12 representa un intento por dar solución a los repetidos fracasos de los sistemas de compensación basados en el mercado o sancionados por el Estado. Esta política propone una metodología de compensación que protege a las poblaciones desplazadas del empobrecimiento inherente a estos procesos. 
El principio básico de la política es bastante directo. Ella requiere que la institución a cargo del proyecto compense a las personas desplazadas: aquellas que han perdido activos económicos, vivienda, fuentes de ingreso o medios de vida como resultado de una adquisición de tierras relacionada con un proyecto o intervención que no ha sido fruto de su elección libre e informada, de manera que su estándar de vida subsecuente sea mejor o por lo menos igual a su situación anterior al desplazamiento. Esto representa el fundamento mínimo de una conducta socialmente responsable en el desarrollo de proyectos de reasentamiento. Para conseguir este objetivo, la política requiere que la institución diseñe un Plan de Reasentamiento específico al caso de intervención en el que se incluya una estrategia de compensación sensible a la realidad y necesidades locales. Dicha estrategia debe ser desplegada a modo de proyecto participativo de desarrollo de tal manera que se permita a las personas desplazadas reconstruir y mejorar sus medios económicos de vida (Cernea, 1997; Cernea, 1999)

$\mathrm{Al}$ ser este un caso de reasentamiento motivado por la llegada de un proyecto de explotación minera, el proceso tiene características que lo diferencian del marco general de desplazamiento por desarrollo (DIDR). El desplazamiento inducido por minería (MIDR, por sus siglas en inglés) tiene sus propias particularidades, que requieren de un análisis más fino para evitar efectos perniciosos. Una característica sobresaliente es que el desplazamiento puede ocurrir en cualquier momento del ciclo de vida del proyecto minero: exploración, construcción u operación. Debido a ello, el proceso de planificación y ejecución del reasentamiento puede quedar a cargo de múltiples equipos de personas con distintos niveles de experiencia en cuestiones sociales (Owen y Kemp, 2015: 481). Asimismo, en los casos de reasentamiento por minería existe la tendencia a la cohabitación y proximidad al sitio de extracción entre las empresas y poblaciones locales. Esto ocurre debido a que, a diferencia de otros proyectos como represas o carreteras, el lugar de intervención atrae actividad económica externa de la cual la población local podría favorecerse (Owen y Kemp, 2015: 482), ya sea a través de la provisión de servicios, empleo directo u otros beneficios. Finalmente, existe una interdependencia (Owen y Kemp, 2015: 484; Ballard y Banks, 2003) entre comunidad y empresa que en otros sectores no se aprecia con la misma frecuencia, pues en estos casos las compañías suelen actuar como mediadoras entre Estado y comunidad a lo largo de extensos procesos de negociación multi-etapa.

\section{El caso de la reubicación de Yanacancha}

El proyecto minero de Antamina está ubicado en el distrito de San Marcos, provincia de Huari, departamento de Ancash. La mina se encuentra en el Callejón de Conchucos, entre la Cordillera Blanca y la Cordillera Huayhuash, a 35 kilómetros del centro poblado de San Marcos (2970 m.s.n.m.), a 165 kilómetros de Huaraz. El depósito de mineral principal yace a 4300 m.s.n.m. La etapa de construcción de la mina empezó en la segunda mitad del año 1998 y las operaciones de extracción hicieron lo propio en el 2001. En agosto de 1998, el yacimiento fue estimado en 500 millones de toneladas comprobadas, con grados de $1.2 \%$ de cobre, $1.1 \%$ de zinc, 15 gramos de plata por toneladas y $0.03 \%$ de molibdeno. El tiempo de vida de la mina es de 40 años desde la puesta en marcha de la extracción. La operación consiste en una mina a "cielo abierto" y una planta concentradora de 70 mil toneladas métricas que producirá 1.3 millones de toneladas de mineral concentrado. Antamina representa una de las más grandes exportadoras del país, con un estimado de 950 millones de dólares norteamericanos al año.

\section{El proceso de reubicación}

Durante el segundo trimestre de 1999, la minera Antamina inició la implementación de un plan de reubicación para las familias del fundo Yanacancha y usufructuarios de los fundos aledaños de Juprog y Tranca - Antamina (en adelante incluidas en el reasentamiento de Yanacancha). Este plan fue modificado 
de un marco general previo debido a los constreñimientos de tiempo a los que el proyecto se encontraba sujeto. Debido a ellos, la compañía adoptó un Plan Acelerado de Reasentamiento (PAR) que incluyó a un total de 53 familias (Glave y Damonte, 2002). Así, el PAR tuvo dos componentes básicos: la introducción de un componente monetario como compensación por el desplazamiento de cada una de las 53 familias, con el objetivo de acelerar el proceso físico de desplazamiento, y un plan de seguimiento y monitoreo para cada una de ellas.

La compañía minera Antamina necesitaba reasentar a las familias campesinas que vivían en el poblado de Yanacancha debido a que, de acuerdo con los planos del proyecto final, la planta concentradora estaría ubicada ahí. Yanacancha era una comunidad altoandina dedicada a la ganadería. Los propietarios de la tierra de Yanacancha eran familias extendidas que en la mayoría de los casos residían en los pueblos ubicados en la parte baja del valle, donde el clima es más cálido. Así, Yanacancha se encontraba permanentemente habitada sólo por familias pastoras dedicadas a cuidar de su ganado ovino.

FIGURA 1

Ubicación del caserío de Yanacancha

MAPA DE UBICACIÓN DEL CASERÍO DE YANACANCHA
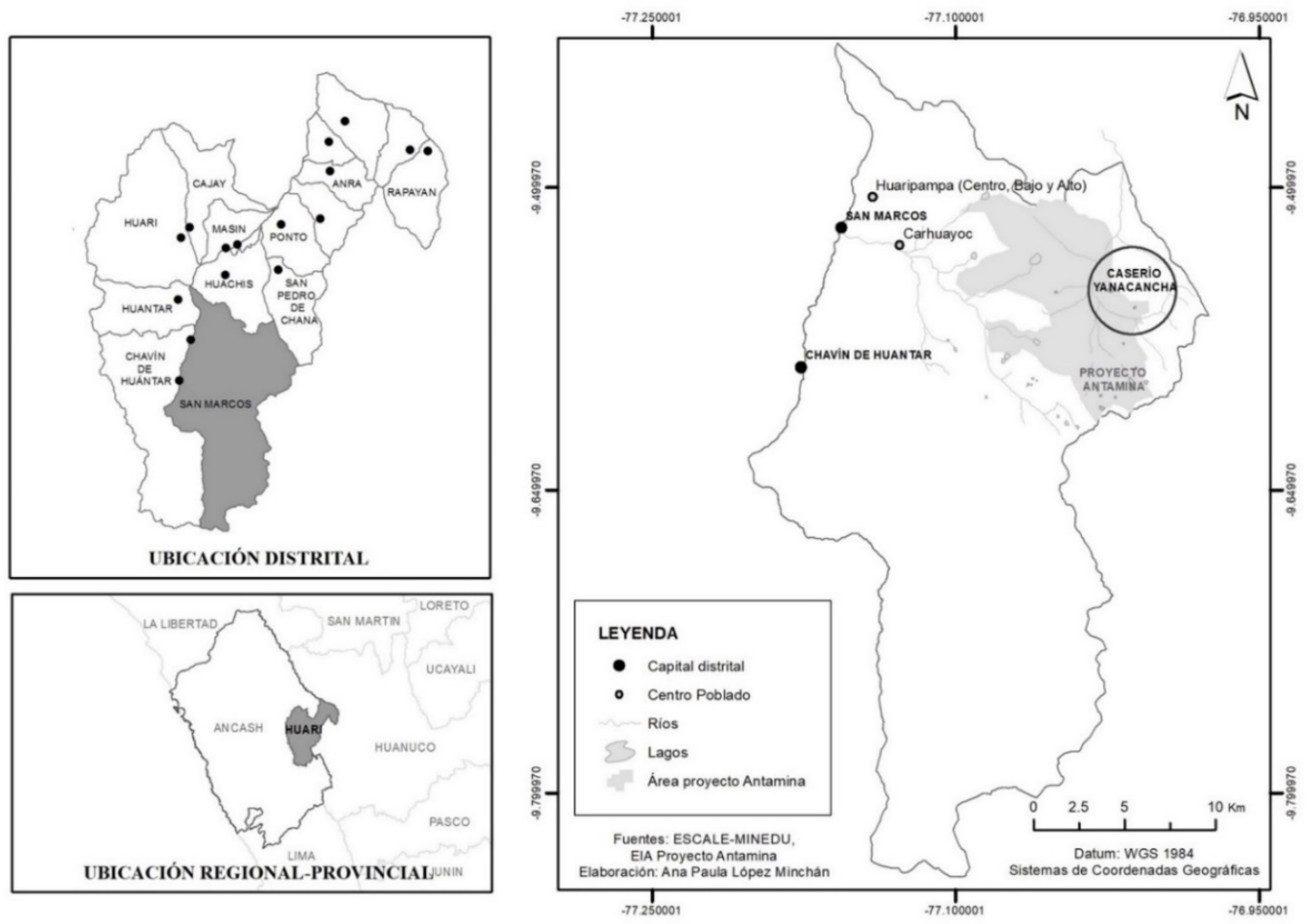

Fuente: Elaboración propia

Típicamente, las familias extendidas andinas tienen y producen a partir de varias parcelas de tierra extendidas en diferentes altitudes del valle, con el objetivo de diversificar y maximizar su producción al reducir el riesgo (Mayer, 2002). En los pastos de altura es costumbre que los propietarios establezcan arreglos con los pastores, quienes pueden o no ser sus familiares, para que se encarguen de los animales, usualmente vacas u ovejas. A modo de pago, las familias pastoras obtienen el derecho de usar los pastos para su propio ganado, que generalmente constituye la mitad del ganado ovino recién nacido de los propietarios.

Sin embargo, la tradición incluye algún grado de participación de los propietarios en las actividades de pastoreo. Las familias extendidas de los propietarios suelen instalarse temporalmente por dos o tres meses 
en las estancias de altura para ayudar o reemplazar a los pastores. Asimismo, los propietarios los visitan regularmente en sus estancias para proveer a los pastores de víveres y otras necesidades, de manera casi ritualizada mediante el intercambio de "cariño" (contraprestación en especies). Los arreglos tradicionales, de este modo, no hacen uso de contratos legales en un sentido occidental, pues se apoyan en relaciones de reciprocidad en las que la responsabilidad y la producción son compartidas. Estas relaciones de reciprocidad se mantienen incluso si la familia extendida crece y se dispersa. En efecto, que algunos miembros se alejen de la puna para estudiar o trabajar en otras ciudades, no significa que dejen de estar vinculados a ella y a la familia mediante, por ejemplo, la propiedad de algunos animales que se quedan al cuidado de sus familiares (Salas, 2008).

A pesar de estas especificidades culturales, la compañía minera diseñó un Plan de Reasentamiento simple y basado en criterios de residencia. Tras adquirir la tierra de los propietarios identificados, la compañía realizó un censo de población en Yanacancha para establecer el número de familias residentes en el área. Se llegó a la cifra de 53 familias, la mayoría pastores que se encontraban presentes en el momento del censo. Habiendo pagado a los propietarios por sus tierras, la minera Antamina consideró a estas 53 familias como las únicas con derecho a participar en el plan de reasentamiento.

Debido a la premura del tiempo, el primer borrador del Plan de Reasentamiento que incluía largos procesos participativos de compensación tierra-por-tierra fue reemplazado por un Plan Acelerado de Reasentamiento (PAR). El PAR mantenía que las 53 familias presentes durante el censo eran las que constituían el universo de la población a reasentar, pero se hicieron algunos cambios en las opciones de compensación y en los procesos de participación para cumplir con los cronogramas del proyecto. En primer lugar, se descartó la opción de compensación tierra-por-tierra debido a que era muy difícil encontrar tierras disponibles en las zonas aledañas a Yanacancha. Luego, se relajaron y aceleraron los procedimientos de participación.

La Compañía Minera Antamina calculó una suma fija de US\$30 000 para cada familia a modo de paquete de compensación por reasentamiento. Esta cantidad cubriría tanto los activos familiares perdidos (principalmente vivienda y ganado) como los gastos de reasentamiento. Las familias podrían elegir su nuevo lugar de residencia. Adicionalmente, la compañía ofreció dos opciones monetarias para hacer entrega de este paquete a las familias

Este plan se llevó a cabo entre marzo y abril de 1999. La idea principal era convencer a las familias afectadas para que se movieran a residencias temporales mientras la mina buscaba predios de reubicación y construía viviendas en ellos. Para esto se elaboraron tres alternativas. La primera opción fue mover a las familias a una gran casa en San Marcos y darles un estipendio de US\$200 al mes para comida por un período de hasta seis meses. La segunda opción consistió en moverse a una casa pre-fabricada construida por la mina en San Marcos, con un estipendio de US\$200 al mes para comida por un período de hasta seis meses. La tercera opción consistía en dar un estipendio de US\$ 500 mensuales por un período máximo de seis meses por gastos de alojamiento y comida.

La totalidad de familias afectadas aceptaron la tercera opción; sin embargo, se mostraron reticentes a moverse de la zona únicamente con la promesa de reubicación. Para superar esta desconfianza, se presentaron dos propuestas a las familias reubicadas. La primera propuesta era brindar un seguro de US\$30 000 (precio calculado para la compra de la tierra y construcción de la vivienda), que sería depositado en el banco a nombre del afectado. Si al cabo de seis meses la mina no cumplía con la reubicación, dicha suma pasaba a disponibilidad del afectado. La alternativa fue depositar la suma de US\$30 000 de inmediato por concepto de reubicación, asegurando ayuda técnica posterior al afectado para establecerse en alguna actividad sostenible.

Las familias se inclinaron por la segunda opción, de tal manera que la reubicación tierra por tierra fue reemplazada por una compensación en efectivo. Entre los meses de marzo y abril de 1999 la Compañía Minera Antamina firmó convenios de reubicación con las 53 familias que consideraba reubicables, las cuales habían sido identificadas en el censo realizado al inicio del proceso. En total fueron 68 familias nucleares las 
que recibieron algún monto de dinero, debido a los problemas en la asignación de derechos de reubicación. Adicionalmente, la mina se comprometió a llevar a cabo un Programa de post-reubicación.

El proceso participativo llevado a cabo por la minera Antamina fue de alcance muy limitado, lo que reflejaba las exigencias de tiempo. Antes de adoptar el PAR, la compañía contactó a los propietarios de Yanacancha y realizó contratos individuales con ellos. Estas personas estaban, por lo tanto, conscientes de la necesidad del reasentamiento por parte de la compañía. Además de esto, la compañía se reunió con líderes comunales para discutir la compra de tierras y el futuro reasentamiento. Sin embargo, este proceso de consulta se encontraba todavía en marcha cuando el PAR fue implementado de manera precipitada.

El PAR fue llevado a cabo en 90 días en 1999. Los depósitos se hicieron a nombre de los jefes de las 53 familias y rápidamente fueron desalojados de sus hogares en Yanacancha, los cuales fueron inmediatamente incendiados. La mayor parte de las familias permanecieron alojadas donde familiares mientras buscaban un lugar dónde instalarse. La compañía brindó asistencia durante el proceso de mudanza.

\section{Protestas e investigación}

Como resultado de los cambios realizados al plan de reubicación para acelerar su ejecución y adecuarse a las exigencias del cronograma establecido, la Compañía Minera Antamina descuido aspectos cruciales de la planificación y cumplimiento de los estándares del Banco Mundial para este tipo de proyectos. La puesta en marcha del PAR en el año 1999 trajo consigo la consecuente confusión del proceso de compras de tierras con el de reubicación, diferencia que nunca quedó clara entre la población afectada. A fin de facilitar la compraventa de las tierras con algunos propietarios que presentaron oposición a las transacciones, se suscribieron los "contratos de reconocimiento de obligaciones" (CRO) con el objetivo de resolver estas oposiciones, con lo que se amplió el número de casos que se consideraban reubicables a pesar de no tener residencia permanente en la zona alta.

Un año antes de la ejecución del PAR, en marzo de 1998, el Proyecto Especial de Titulación de Tierras (PETT) del Ministerio de Agricultura presentó los resultados de su trabajo y se inició el proceso de adquisición de tierras. El precio de las tierras se acordó luego de negociaciones individuales sostenidas por los propietarios con la empresa minera. Así, se estableció el precio de US\$1000 x hectárea en los fundos de Antamina y Tranca, y US\$ $400 \mathrm{x}$ hectárea en el resto de los fundos; estos valores eran muy superiores a los que usualmente se pagan en tierras de altura en la sierra peruana y al "justiprecio" que establece el Ministerio de Agricultura. En los casos en que los propietarios presentaron oposición a la compra-venta, la minera optó por comprometerse a brindarles otro tipo de compensaciones a través del futuro Plan de Reubicación por medio de Contratos de Reconocimiento de Obligaciones (CRO). De acuerdo con estos documentos, los propietarios que firmaban un CRO contaban con el compromiso de la minera para ser considerados reubicables dentro del Proceso de Reubicación. El contenido de estos documentos generó dificultades tanto para el cierre legal del proceso de reubicación como para el cierre social de todo el proceso de compra de tierras y reubicación de las familias residentes.

Así, varios propietarios que no habían presentado oposición y, por lo tanto, no habían negociado la firma de un CRO con la compañía, más allá del tipo de residencia que habrían tenido en la parte alta, presentaron quejas o reclamos posteriormente. En general, estos compromisos ya se habían cumplido a través de Transacciones Extrajudiciales, pero quedaban pendientes aún unos 4 casos, entre los que se incluía la Comunidad Campesina de Ango Raju.

La implementación del PAR desencadenó una oleada de protestas en la localidad. Por un lado, aparecieron varias personas que reclamaban ser propietarias, afirmando sus derechos sobre la tierra adquirida. Por el otro, llegaron residentes temporales y permanentes que no se encontraban en el momento del censo, quienes protestaron por no ser reconocidos como familias afectadas. Este reclamo venía de parte de propietarios residentes y miembros ancianos o muy jóvenes de las familias extendidas que no habían sido incluidos en las 
negociaciones. En las comunidades vecinas se replicó el descontento y siguieron protestas por no haber sido consultados sobre la implementación del PAR.

Eventualmente, las quejas locales llegaron a audiencias internacionales, por lo que el Banco Mundial decidió enviar un comité investigador (Van Veldhuizen y Tarazona, 2000) de la agencia internacional MIGA (Multilateral Investment Guarantee Agency). El comité encontró algunas irregularidades en el proceso y en su informe consideró que un problema recurrente en la intervención liderada por minera Antamina fue la percepción negativa de la población hacia el proceso de reubicación. Esta percepción negativa se tradujo en desconfianza y suspicacia, que predispusieron a la población a recelar de cualquier nueva propuesta de la empresa. Para encontrar solución a estos problemas, la comisión sugirió que la compañía realizara una evaluación independiente del PAR y cumplir con los estándares del Banco Mundial sobre reasentamiento. Tanto la compañía como el Banco Mundial estuvieron de acuerdo en encargar a GRADE esta evaluación independiente en el año 2000.

La evaluación de GRADE encontró serios problemas con el diseño e implementación del PAR. Las dos principales cuestiones fueron la incomprensión de los patrones de producción y la residencia de las familias campesinas andinas (Glave y Damonte, 2002). En primer lugar, el PAR no tomó en cuenta las especificidades sociales y culturales que complejizan el criterio de residencia permanente, favorecido por la minera para determinar a los participantes del reasentamiento. Siguiendo pautas culturales, los hogares involucrados mantenían más de una morada, mudándose estacionalmente a otros lugares para vigilar sus cultivos o rebaños. Asimismo, muchos hogares estaban compuestos por más de una familia nuclear. La propiedad y uso de la tierra corresponde a la familia extendida como totalidad, aun cuando cada familia nuclear tiene responsabilidades específicas, como pastorear a los animales.

Las políticas del Banco Mundial no son específicas sobre este asunto, así que la compañía identificó de manera binaria a residentes y no-residentes, con lo que ocultaba las dinámicas andinas de producción familiar. Asimismo, la empresa no identificó a los desplazados económicos, es decir, a aquellos que son afectados pero no necesariamente tienen que salir del lugar original.

El PAR privilegió las negociaciones con familias individuales por sobre la consulta al colectivo comunal. Al hacer esto la compañía obvió dos espacios sociales fundamentales para la toma de decisiones sobre el territorio: las familias extendidas y las comunidades campesinas. La compañía minera debió haber reconocido que las familias pastoras de Yanacancha eran parte de las familias extendidas que vivían en distintas partes del valle y formaban parte de comunidades campesinas. Dados los acuerdos de producción recíprocos entre los miembros de una familia extendida, debió haber estado claro que, si los pastores eran desplazados, esto afectaría de una u otra manera al resto de los miembros de la familia extendida y comunidades de pertenencia. De hecho, muchas familias de pastores que eran parte de la comunidad vecina de Angoraju Carhuayoc luego de la reubicación se asentaron el centro poblado de dicha comunidad (Damonte, 2008).

En resumen, la aplicación de las políticas de reubicación en el caso de PAR fue deficiente en dos temas: la adecuada identificación de las personas y colectivos afectados y la identificación de los representantes válidos de dichos colectivos. En la siguiente sección, a partir del estudio de GRADE, explicamos la importancia de ajustar los mecanismos de política al contexto alto-andino con el fin de identificar adecuadamente a las personas y colectivos afectados, así como establecer sus distintos grados de afectación en el contexto de sistemas de producción andinos. 


\section{3. ¿Cómo generar una intervención Culturalmente sensible? Propuestas METODológicas a PARTiR de LA EVALUACión DEL PAR}

\section{Identificación de afectados}

La propiedad de la tierra muchas veces define la forma de producción y el beneficio indirecto. En Yanacancha, los propietarios que no eran residentes mantenían en la mayoría de los casos algún tipo de lazo con los residentes permanentes. Estos lazos servían de vehículo para el acceso indirecto a recursos por parte de los propietarios y se establecían a través de lazos de reciprocidad que podían o no implicar parentesco sanguíneo o ceremonial. Por ello, el PAR afectaba no sólo a los residentes sino también a los propietarios no residentes, muchos de los cuales no fueron tomados en cuenta (Salas, 2008). Así, para identificar las redes de parentesco existentes en Yanacancha y de tal forma identificar quiénes serían mayormente afectados, GRADE realizó un ejercicio de investigación paralelo que consistió en la construcción de árboles familiares teniendo como punto de partida a los propietarios más antiguos de los fundos de Yanacancha y Tranca / Antamina. En estos árboles se puede observar no sólo la conformación de las familias en cinco siblings sino que también se identifica a los individuos que fueron compensados de alguna manera durante el PAR, así como a los que han presentado reclamos por reubicación a la empresa.

En relación con los grados de afectación, ya que los propietarios mantenían varios tipos de usufructo de sus recursos en la parte alta, GRADE realizó una categorización a partir del grado de residencia en aquella zona. Aunque el grado de residencia no determina necesariamente el grado de importancia de los recursos en la zona alta para cada uno de los propietarios (recordemos que "propietarios" implica colectivos y no individuos), creemos que sirve como un indicador ya que implica una mayor dedicación en términos de tiempo asignado. En primer lugar, los residentes que manejaban directamente sus recursos. En este caso, el proceso de compra y reubicación afectó una gran parte de su economía. No podemos afirmar que se trata de la totalidad de sus fuentes de ingreso porque las redes que se mantienen con familiares y/o comuneros al interior de su colectivo fluían en ambos sentidos, haciendo que estos residentes tuvieran acceso indirecto a recursos en otras tierras. Sin embargo, debemos señalar que el reemplazo o sustitución de sus recursos por un stock de capital financiero debilitó de manera ostensible su posición en la red (parental o comunitaria) puesto que perdieron su capacidad de replicar el flujo de recursos.

En segundo lugar, los usufructuarios indirectos - residentes rotativos. En este caso los usufructuarios no son residentes permanentes; sin embargo, su base económica la constituyen el ganado y los cultivos que mantenían en la parte alta. En tercer lugar, los usufructuarios indirectos con residencia estacional. Se trataba de los propietarios que suben de manera esporádica a vigilar sus tierras y/o ganado a la parte alta con el fin de asegurarse el buen mantenimiento de sus recursos. En estos casos, el propietario contrata o se asocia con un residente permanente, a quien le encarga el manejo cotidiano de su ganado o cultivo. Los usufructuarios indirectos estacionales suben para restablecer los lazos con sus pastores a partir de arreglos tradicionales y/o participan en la faena de siembra y cosecha de las tierras cultivables.

En cuarto lugar, los usufructuarios indirectos no-residentes. Típicamente, hablamos de segmentos de familias extensas propietarias que han optado por no mantener ninguna presencia física en las tierras. Sin embargo, mantienen participación en el ganado y/o cosechas derivadas del uso de las tierras. Estos propietarios mantienen niveles de dependencia económica con la tierra mucho menores, pero siguen siendo parte del sistema de producción y uso de recursos. Finalmente, los campesinos sin derecho legal sobre la tierra o precarios, que mantienen un sistema de acceso a recursos similar al de los propietarios y muchas veces forman parte de los mismas redes familiares de los primeros aunque no tengan ningún arreglo "formal". 


\section{Identificación de grados de afectación y compensación}

Para determinar los derechos de reubicación de las familias se utilizaron dos criterios de Unidad familiar: a partir de los árboles de parentesco se estableció el universo de familias afectadas, y Fogón: a partir de la identificación de los espacios de preparación y consumo de alimentos de la familia se establecieron los espacios de residencia. Estos criterios fueron luego complementados con otras consideraciones referidas a la vulnerabilidad de las familias y al tiempo de residencia en la puna (para aquellos que llegaron recientemente, así como para aquellos casos de 'mala suerte' que se acaban de retirar voluntariamente de la puna). A partir de esta clasificación se establecieron tres tipos de residencia. Primero, residencia permanente: aquellas familias que vivían todo el año en la puna con muy poca conexión productiva con las tierras bajas; un indicador importante de estas familias, en el caso de Yanacancha, es que sus hijos menores de edad iban a la escuela de Yanacancha. Segundo, residencia temporal (o rotativa): aquellas familias que 'cambiaban' o rotaban el cuidado de estancias y ganado; el período de permanencia permanente en la puna era casi continuo en la época de lluvias (cuando aumenta la cantidad de pastos), y más esporádico y rotativo en la época seca. Tercero, residencia estacional: aquellas familias que tenían ganado encargado y subían solamente en la época de siembra y cosecha de sembríos.

A partir de esta clasificación, el estudio de GRADE (Glave y Damonte, 2000) identificó hasta cinco tipos de impacto y paquetes de compensación correspondientes a cada uno. Especial atención se les prestó a los casos de hogares vulnerables, es decir, aquellos con ancianos o personas con discapacidad sin acceso a seguros sociales (ver Tabla 1).

TABLA 1

Tipología de grados de afectación

\begin{tabular}{|c|c|c|c|c|c|}
\hline Afectación & $\begin{array}{l}\text { I derecho a } \\
\text { Con por } \\
\text { compensación } \\
\text { tierras y reubicación }\end{array}$ & $\begin{array}{l}\text { II } \\
\text { Con derecho a } \\
\text { compensación sólo por } \\
\text { reubicación; igual que el } \\
\text { afectado I pero sin } \\
\text { derechos de propiedad }\end{array}$ & $\begin{array}{l}\text { III } \\
\text { Con derecho a } \\
\text { compensación por tierras o } \\
\text { reubicación y algo más } \\
\text { (vivienda, ganado, } \\
\text { vulnerabilidad); tienen } \\
\text { derecho a reubicación } \\
\text { familiar (extensa), colectiva. } \\
\text { Son ejemplos claros de } \\
\text { desplazamiento de unidades } \\
\text { económicas }\end{array}$ & $\begin{array}{l}\text { IV } \\
\text { Con derecho a } \\
\text { compensación sólo por } \\
\text { tierras; derecho a que le } \\
\text { compren el ganado a } \\
\text { precio alzado }\end{array}$ & $\begin{array}{l}\text { Con derecho a } \\
\text { alguna } \\
\text { compensación que } \\
\text { no es ni por tierras } \\
\text { ni por reubicación }\end{array}$ \\
\hline & $\begin{array}{l}\text { - Propietario } \\
\text { - Con residencia tipo j, } \\
\text { fogón independiente } \\
\text { en la parte alta y } \\
\text { familia constituida (1- } \\
1-1) \\
\text { - Posesión y/o } \\
\text { usufructo de ganado } \\
\text { y/o tierras cultivables } \\
\text { - Puede o no ser un } \\
\text { caso de vulnerabilidad } \\
\text { - Indicadores } \\
\text { adicionales de acceso } \\
\text { a recursos alternativos }\end{array}$ & $\begin{array}{l}\text { - Con residencia tipo i, } \\
\text { fogón independiente } \\
\text { en la parte alta y } \\
\text { familia constituida (1- } \\
\text { 1-1) } \\
\text { - Posesión y/o } \\
\text { usufructo de ganado } \\
\text { y/o tierras cultivables } \\
\text { - Puede o no ser un } \\
\text { caso de vulnerabilidad } \\
\text { - Indicadores } \\
\text { adicionales de acceso } \\
\text { a recursos } \\
\text { alternativos }\end{array}$ & $\begin{array}{l}\text { - Propietario o precario } \\
\text { - Residencia "rotativa } \\
\text { (temporal) (tipo ii), con } \\
\text { fogón compartido y familia } \\
\text { constituida }(2-1-1) \\
\text { - Puede o no ser un caso de } \\
\text { vulnerabilidad } \\
\text { - Indicadores adicionales de } \\
\text { acceso a recursos } \\
\text { alternativos }\end{array}$ & $\begin{array}{l}\text { - Propietario } \\
\text { - Residencia estacional } \\
\text { (tipo iii), sin fogón en la } \\
\text { parte alta } \\
\text { - No tiene activos físicos } \\
\text { importantes (casa, canal, } \\
\text { etc.) } \\
\text { - Ganado manejado por } \\
\text { otra familia en una } \\
\text { majada aparte } \\
\text { - Puede o no ser un caso } \\
\text { de vulnerabilidad }\end{array}$ & $\begin{array}{l}\text { - Precario } \\
\text { - Con acceso } \\
\text { indirecto a } \\
\text { ganado, uso de } \\
\text { tierras, y hasta } \\
\text { vivienda } \\
\text { (almacén) } \\
\text { - Residencia } \\
\text { estacional (tipo } \\
\text { iii) }\end{array}$ \\
\hline
\end{tabular}


Esta escala nos aproxima al grado de afectación por familia luego del PAR. Sin embargo, la empresa minera brindó un conjunto de compensaciones, ya sea por tierra o por reubicación tanto por vía legal como extrajudicial. Estas compensaciones pueden ser a su vez catalogadas a fin de comparar el grado de afectación con la compensación recibida. Los tipos de compensación pueden ser catalogados como se presentan en la Tabla 2.

TABLA 2

Tipología de compensaciones

\begin{tabular}{|c|c|c|c|c|}
\hline $\begin{array}{l}\text { Compen- } \\
\text { sación }\end{array}$ & I & II & III & IV \\
\hline & $\begin{array}{l}\text { - Recibió dinero por } \\
\text { venta de tierras } \\
\text { - Recibió dinero por } \\
\text { PAR } \\
\text { - Recibió asistencia / } \\
\text { apoyo post PAR } \\
\text { - Puede haber } \\
\text { recibido } \\
\text { compensación por } \\
\text { algún activo } \\
\text { especifico) }\end{array}$ & $\begin{array}{l}\text { - Recibió dinero } \\
\text { por } \\
\text { reubicación } \\
\text { - Recibió } \\
\text { asistencia / } \\
\text { apoyo post } \\
\text { PAR }\end{array}$ & $\begin{array}{l}\text { - Recibió dinero } \\
\text { por venta de } \\
\text { tierras } \\
\text { (durante la } \\
\text { compra o } \\
\text { extrajudicial) }\end{array}$ & $\begin{array}{l}\text { - Recibió } \\
\text { compensación } \\
\text { por algún } \\
\text { activo } \\
\text { (vivienda o } \\
\text { ganado) }\end{array}$ \\
\hline
\end{tabular}

Fuente: Glave y Damonte 2000

Este ejercicio nos da un punto de partida para establecer grados de afectación no compensados satisfactoriamente o simplemente no compensados en absoluto. Sin embargo, teniendo en cuenta que los lineamientos del trabajo precisan que, más allá de los grados de afectación y la compensación recibida, las familias deben lograr un nivel de vida superior o al menos similar al que tenían antes de la llegada de la Compañía Minera Antamina, se completó el análisis con la introducción de una variable adicional, como es la del monitoreo de las condiciones de vida luego del proceso de reasentamiento.

\section{Conclusiones}

El análisis nos muestra que, para mejorar la implementación de las políticas de reubicación, estás deben contar con un diseño que les permita ajustarse a los contextos culturales, económicos y políticos específicos de la población y su territorio (Escobar, 2001; Agrawal, 1999; Agrawal y Redford, 2009). En el caso de Yanacancha queda claro que la implementación de la reubicación no tomó en cuenta las características de los sistemas de producción pastoriles que permitían la reproducción social y económica de la población a reubicar (Salas, 2008; Gil, 2009). Por ello, la reubicación afectó de manera significativa dichos sistemas, sin establecer mecanismos para ayudar a su reconstrucción social. Como resultado, la política no pudo lograr uno de sus objetivos centrales: la reconstrucción social de la población reubicada. 
Los sistemas de producción pastoriles están caracterizados por ser una adaptación cultural de las condiciones ecológicas altoandinas, un ecosistema de pastizales en tierras semiáridas poco adecuado para la agricultura pero funcional para la alimentación de camélidos andinos. El pastoralismo se apoya en una estrategia doble de especialización y articulación (Flores Ochoa, 1975; Browman, 1974; Custred, 1977). La especialización implica maximizar los forrajes locales para la alimentación del ganado mientras la articulación involucra mantener lazos económicos con valles agrícolas para obtener otros productos.

Gracias a estos lazos entre distintas zonas los pastores obtienen la capacidad de residir en las estancias de alta montaña por largos períodos. Estas estrategias requieren que los pastores posean un alto grado de movilidad, característica que muchos autores consideran como el principio alrededor del cual se organizan las familias pastoriles (Custred, 1977; Medinaceli, 2005). La movilidad de los pastores está regulada por un patrón de usos de recursos conformados por cambios estacionales según la disponibilidad de pasturas, así como por los ciclos diarios y anuales del manejo de rebaños (Damonte, Glave, Kiriki, Njagi, Rodríguez, 2019). Esta especificidad cultural y territorial de las comunidades pastoriles andinas se enmarca en formas culturalmente definidas de producción en los Andes, conocidas hace décadas como el control vertical de un máximo de pisos ecológicos (Murra, 1975).

A partir de estas particularidades de la organización económica y social de las familias pastoras de montaña, se hace patente la necesidad de contar con una tipología de sistemas de producción para evitar errores típicos, como pasar por alto la importancia de las familias extendidas y otras formas de acción colectiva en el manejo de la tierra, pastizales y otros activos. Así, el grado de impacto no se debe definir únicamente como la pérdida de activos físicos como la tierra, pastizales, viviendas y ganado, sino también como la pérdida de redes sociales y la habilidad de interactuar dentro de un sistema social que permite el acceso a un conjunto de recursos o activos. En otras palabras, a largo plazo, el grado de impacto debería evaluarse en términos de la reducción de la capacidad de reproducción de una serie de activos siguiendo un conjunto claramente definido de reglas económicas de acceso y uso de estos recursos.

Con respecto al paradigma de la participación informada, el caso de Yanacancha claramente demostró que cualquier Plan de Reasentamiento en un ecosistema de alta montaña no puede obviar la dimensión de la acción colectiva en los sistemas económicos y sociales originales. La forma apropiada de participación comunal en los planes de reasentamiento debe estar diseñada en diálogo con las comunidades en cuestión. Esto podría traducirse en un ajuste de política para asegurar la representación de las comunidades y otros colectivos en el proceso de planificación de un reasentamiento.

Asimismo, los procesos de reasentamiento inducidos por minería, a diferencia de otros desplazamientos por proyectos no productivos, deben tener en cuenta la experiencia de las comunidades afectadas con industrias extractivas (Miranda, 2016; Damonte, 2008) y su grado de inclusión en sistemas económicos monetizados. El caso de Yanacancha muestra que, en una sociedad pastoril de alta montaña con un limitado acceso a la economía comercial, el proceso de monetización no puede reemplazar satisfactoriamente los procesos de acumulación de activos en forma de ganado o tierra si la compensación se limita solamente a una inyección de recursos financieros en la forma de flujos de ingresos de corto plazo. Debido a esto, queda claro que la reconstrucción de los medios de vida en sociedades pastoriles de alta montaña requiere de formas de compensación tierra-por-tierra.

Finalmente, consideramos que hay una dificultad metodológica en la implementación de los estándares internacionales como la Política Operacional 4.12 del Banco Mundial en el caso de ecosistemas de montaña. Claramente, lo que se requiere es una clasificación del grado de impacto en el conjunto de familias antes que una relación de "familias aptas" (basada en un criterio ineficiente de residencia permanente) para diseñar cualquier plan de reasentamiento. La principal lección que nos deja el caso de Yanacancha, más allá de los dilemas de los derechos colectivos no formales tradicionales en sociedades tradicionales (Szablowki, 2002), es la necesidad de un diseño de política que tome en cuenta el territorio donde se aplique, es decir, 
cuya implementación esté basada en las características culturales, políticas y económicas del espacio de intervención.

\section{ReFERENCIAS}

Agrawal, A. (1999). Enchantment and Disenchantment: The Role of Community in Natural Resource Conservation. World Development, 27(4), 629-649.

Agrawal, A. y K. Redford (2009). Conservation and Displacement: An Overview. Conservation and society, 7(1), 1-10.

Ballard, C., y G. Banks (2003). Resource Wars: The Anthropology of Mining. Annual Review of Anthropology, 32, 287-313.

Banco Mundial (1986). Operations policy issues in the treatment of involuntary resettlement. Operational Policy Note 10.08. Washington DC: World Bank.

Banco Mundial (2004). Involuntary Resettlement Sourcebook. Planning and Implementation in Development Projects. Washington DC: World Bank.

Barca, F., McCann, P. y Rodríguez-Pose, A. (2012). The Case for Regional Development Intervention: Place-Based Versus Place-Neutral Approaches. Journal of Regional Science, 52(1), 134-152.

Browman, D. (1974). Pastoral Nomadism in the Andes. Current Anthropology, 15(2), 188-196.

Cernea, M. (1988). Involuntary Resettlement and Development. Finance \& Development, 25(3), 44-46.

Cernea, M. (1997). The Risks and Reconstruction Model for Resettling Displaced Populations. World Development, Vol. 25, No. 10, 1569-1587.

Cernea, M. (Ed.) (1999). The economics of involuntary resettlement. Washington DC: World Bank.

Cernea, M. (2000). Risks, safeguards, and reconstruction: a model for population displacement and resettlement. En M. Cernea, y C. McDowell (Eds.). Falta el número de pp. Risks and Reconstruction. Experiences of Resettles and Refugees. Washington DC: World Bank.

Cernea, M. (2003). For a new economics of resettlement: a sociological critique of the compensation principle. International Social Science Journal, 55(175), 37-45.

Custred, G. (1977). Las Punas de los Andes Centrales. En J. Flores Ochoa (Ed.). Pastores de Puna. Uywamichiq punarunakuna (pp. 55-85). Lima: Instituto de Estudios Peruanos.

Damonte, G. (2008). The constitution of political actors in the Andes. Berlín: VDM Verlag.

Damonte, G., Glave, M., Kiriki, L., Njagi, T. y Rodríguez, S. (2019). Land tenure and the sustainability of pastoral production systems: a comparative analysis of the Andean Altiplano and the East African savannah. Nomadic Peoples, 23(1), 28-54.

Downing, T. (2002). Avoiding New Poverty: Mining-Induced Displacement and Resettlement. Mining, Minerals and Sustainable Development (MMSD), 58. Recuperado de https://pubs.iied.org/pdfs/G00549.pdf

Escobar, A. (2001). Culture Sits in Places: reflections on globalism and subaltern strategies of localization. Political Geography, 20, 139-174.

Escobedo, J. (2008). Sobre la constitucionalidad de la reubicación de poblaciones: a propósito del caso de Cerro de Pasco. Lima: CEPES.

Flores Ochoa, J. (1975). Pastores de Alpacas. Allpanchis, 8, 5-23.

Freeney, P. (1998). Accountable aid. Local participation in major projects. Oxford: Oxfam.

Gil, V. (2009). Aterrizaje minero. Cultura, conflicto, negociaciones y lecciones para el desarrollo minero desde la mineria en Ancash. Lima: Instituto de Estudios Peruanos.

Glave, M. y Damonte, G. (2000). Evaluación del proceso de reubicación y del programa post-reubicación en Antamina. Inédita.

Glave, M. y Damonte, G. (2002). Evaluación independiente del programa de reubicación de la compañia minera Antamina. Lima: GRADE. 
Hervé, B. (2013). En attendant d'être réinstallés: discours et conflit autour des enjeux du déplacement d'une communauté paysanne au Pérou. Autrepart, 64, 71-88.

Kanbur, R. (2003). Development economics and the compensation principle. International Social Science Journal, 55(175), 27-35.

León, C. (2015). Reubicación de poblaciones por proyectos mineros en el Perú. Diferencias por género y edad. En N. Henríquez, G. Damonte, M. Braig y B. Göbel (Eds.). Desigualdades en un mundo globalizado (pp. 201 - 214). Lima: CISEPA, PUCP.

Massey, A. (2009). Policy mimesis in the context of global governance. Policy Studies, 30(3), 383-395.

Mayer, E. (2002). The articulated peasant: household economies in the Andes. Boulder: Westview Press.

Medinaceli, X. (2005). Los pastores andinos: una propuesta de lectura de su historia. Ensayo bibliográfico de etnografía e historia. Bulletin de l'Institut Français d'Études Andines, 34(3), 463-474.

Miranda, F. (2016). Politicas de lugar en Morococha: el contexto del reasentamiento por el proyecto minero Toromocho (Tesis de Licenciatura en Antropología). Lima: PUCP. Inédita. Recuperado de http://hdl.handle.net/20.500. $12404 / 9179$

Murra, J. (1975). Formaciones económicas y politicas del mundo andino. Lima: Instituto de Estudios Peruanos.

McDowell, C. (Ed.) (1996). Understanding impoverishment. The consequences of development-induced displacement. Oxford: Bergham Books.

OECD (2011). OECD Regional Outlook 2011: Building Resilient Regions for Stronger Economies. Recuperado de https://www.oecd-ilibrary.org/urban-rural-and-regional-development/oecd-regional-outlook-2011_97892 64120983-en

Owen, J. y Kemp, D. (2015). Mining-induced displacement and resettlement: a critical appraisal. Journal of Cleaner Production, 87, 478-488.

Price, S. (2009). Prologue: victims or partners? The social perspective in development-induced displacement and resettlement. Asia Pacific Journal of Anthropology, 10(4), 266-282.

Salas, G. (2008). Dinámica social y minería: familias pastoras de puna y la presencia del proyecto Antamina (1998-2002). Lima: IEP.

Syvatera, J. (2016). Creation and Domestication of Global Policy Trends: The case of national bioethics committees. Finland: Tampere University Press. Recuperado de https://trepo.tuni.fi/bitstream/handle/10024/98862/978 -952-03-0098-2.pdf? sequence=1\&isAllowed $=y$

Szablowski, D. (2002). Mining, Displacement and the World Bank: A Case Analysis of Compania Minera Antamina's Operations in Peru. Journal of Business Ethics, 39(3), 247-273.

Szablowski, D. (2007). Who Defines Displacement? The Operation of the World Bank Involuntary Resettlement Policy in a Large Mining Project. En P. Bose, P. Idahosa y P. Vandergeest (Eds.). Development's Displacements: Ecologies, Economies, and Cultures at Risk. (Pp. 33-60). Vancouver-Toronto: UBC Press.

Van Veldhuizen, H. y Tarazona, E. (2000). Peru: Antamina Mine. Report by the MIGA Mission. 